\title{
Optional processes in similarity judgments
}

\author{
ROBERT D. MELARA \\ Purdue University, West Lafayette, Indiana \\ LAWRENCE E. MARKS \\ John B. Pierce Laboratory and Yale University, New Haven, Connecticut \\ and \\ KATHRYN E. LESKO \\ New School for Social Research, New York, New York
}

\begin{abstract}
This research investigates the nature of similarity relations among three pairs of interacting dimensions: (1) the integral dimensions of auditory pitch and loudness, (2) the configural dimensions of paired parentheses, and (3) the cross-modally corresponding dimensions of visual position and auditory pitch. We evaluated the rules by which information from each dimension combines in similarity judgments. Our claim is that, when judging similarity, processes that are obligatory, or what we call mandatory processes, can commingle with processes of choice, or what we call optional processes. By varying instructions, we found strong evidence of optional processing. Instructions to rate overall similarity encouraged subjects to attend to stimuli as wholes and led to a Euclidean rule in similarity scaling. Instructions to focus on dimensions encouraged subjects to consider each stimulus dimension separately and led to a city-block rule. We argue that optional processes may obscure mandatory ones, and so need to be identified before mandatory processes can be understood.
\end{abstract}

What rules govern similarity relations among a set of multidimensional stimuli? The answer to this question has important implications in many subfields of cognitive psychology. In one particular subfield, that concerned with issues of dimensional interaction in perception, this critical question has received some tentative answers. There, a distinction is made between interacting dimensions and separable dimensions (e.g., Garner, 1974b). A pair of interacting dimensions is, theoretically speaking (see Melara \& Marks, 1990a, 1990b, 1990c, 1990d), one characterized by crosstalk: Processing in one channel (dimension) affects concurrent processing in the other channel. Color saturation and brightness exemplify interacting dimensions (Garner \& Felfoldy, 1970). A pair of separable dimensions, on the other hand, is one in which channels of processing are largely autonomous across levels of analysis: Processing in one channel occurs independently of processing in another channel. Circle size and diameter orientation exemplify a separable pair of dimensions (Garner \& Felfoldy, 1970).

The conceptual distinction between interacting and separable pairs of dimensions has been bolstered by a set of

Portions of this research were presented at the 31 st Annual Meeting of the Psychonomic Society in New Orleans, November 1990. Preparation of this paper was supported by National Institutes of Health (NIH) Postdoctoral Fellowship HD07137 and NIH Grant NS28617 to Robert Melara and by NIH Grant DC00271 to Lawrence Marks. We thank Norma Graham and two anonymous reviewers for their careful and helpful comments on an earlier draft. Address all correspondence to Robert D. Melara, Department of Psychological Sciences, Purdue University, West Lafayette, IN 47907. converging operations (Garner, Hake, \& Eriksen, 1956), two of which are discussed in this article. One operation is based on what geometric relations obtain when subjects rate similarity among stimuli formed from either interacting or separable pairs of dimensions. By characterizing psychological stimuli as points in some multidimensional space, one can ask what psychological rule subjects use to compute distances. With separable dimensions, subjects typically rate stimulus similarity by comparing values on each stimulus dimension separately and then summing their judgments. The distance rule used to combine separable dimensions is thought to be the Minkowski cityblock metric:

$$
d_{i j}=\sum_{k=1}^{N}\left|x_{i k}-x_{j k}\right|,
$$

where $d_{i j}$ is the city-block distance between two stimuli, $i$ and $j, x_{i k}$ is the value of stimulus $i$ on dimension $k$, and $N$ is the number of dimensions of difference. The interpoint distances along each of $N$ component dimensions are computed and then summed across $k$. In studies that use techniques of multidimensional scaling (MDS) to analyze similarity judgments, distances among stimuli created from separable dimensions usually are fit best by the city-block metric (Attneave, 1950; Handel \& Imai, 1972; Hyman \& Well, 1967, 1968).

In contrast, a Euclidean metric appears to characterize the similarity space of interacting dimensions:

$$
d_{i j}=\sqrt{\sum_{k=1}^{N}\left|x_{i k}-x_{j k}\right|^{2}} .
$$


MDS investigations have confirmed that the best distance rule for stimuli formed from interacting dimensions is Euclidean (e.g. 2 Handel \& Imai, 1972; Hyman \& Well, 1967, 1968; Indow \& Kanazawa, 1960; Melara, 1989b; Torgerson, 1958). The usual interpretation of this finding is that subjects do not distinguish dimensions when judging similarity, but instead compare stimuli directly on the basis of the perceptual whole (see Garner, 1974b; Smith \& Kemler, 1978). Although not discussed in this article, several other interpretations have been proposed (see Melara \& Marks, 1990c).

Using a geometric model, then, one can capture the possible similarity relations between different pairs of dimensions through the generalized Minkowski distance function,

$$
d_{i j}=\left(\sum_{k=1}^{N}\left|x_{i k}-x_{j k}\right|{ }^{r}\right)^{1 / r},
$$

where the exponent $r$ equals 1 (the city-block metric) when dimensions are separable and equals 2 (the Euclidean metric) when dimensions interact.

\section{Mandatory Versus Optional Processes}

Traditionally, the distance metric obtained in similarity scaling has been thought to reflect the true or perceived structure of a set of stimuli (Garner, 1974b). In this sense, the distance metric-city-block or Euclidean-reflects obligatory or mandatory properties of dimensional processing. Indeed, Shepard (1987) has argued for a universal law of generalization, which states that a fundamental property of generalization phenomena is the metric that structures multidimensional psychological space: Euclidean for stimuli formed from interacting dimensions, cityblock for stimuli formed from separable dimensions (see also Ennis, 1988; Ennis, Palen, \& Mullen, 1988). The generalization or similarity structure is considered inherent in the nature of the dimensions themselves. This is particularly true in the case of interacting dimensions, where subjects are thought not to have easy perceptual access to the component dimensions.

In this article, we ask, To what extent are the distance functions obtained in similarity scaling under optional control? In other words, How much freedom does the subject exercise in determining the rule that structures the similarity space? If similarity judgments reflect only a mandatory similarity structure, then we would expect little success in altering best-fitting distance functions. If, on the other hand, subjects merely adopt a preferential mode of responding, then we would expect the psychological rule to be malleable and, hence, not obligatory.

The distinction between mandatory and optional processes was advanced earlier by Garner (1974a). His treatment was restricted to a logical analysis of possible patterns of performance in certain information-processing tasks. But the distinction between these two processes is a fundamental one of broad generality. In our view, confusing mandatory with optional processes is troublesome for progress in cognitive psychology, because understanding cognitive phenomena may begin only by isolating what subjects must do, not what they choose to do. Operations that give evidence of optional processes may mask mandatory ones, and so need to be identified and isolated.

In this article, we demonstrate that mandatory processes cannot be assumed to dictate the geometry of multidimensional psychological space; similarity ratings can be based largely in optional processes. This research thus fits well with recent evidence suggesting that phenomena alleged to have wholly mandatory origins, such as Stroop interference and Garner interference, can contain large optional components (see, e.g., Melara, 1989a, 1990; Melara \& Marks, 1990d). The role of optional factors in processing similarity relations has been hinted at by evidence on the effects of instructions in MDS (e.g., Fraser, 1976; Parker, Schneider, \& Kanow, 1975; Shepard, 1964; Shepard, Kilpatric, \& Cunningham, 1975). In this article, the optional components of similarity are addressed more systematically than has been the case hitherto.

\section{Types of Interacting Pairs of Dimensions}

We test for optional processes in three different pairings of interacting dimensions: (1) integral dimensions-auditory pitch and loudness; (2) configural dimensions-pairs of parentheses; (3) cross-modally corresponding dimensionsauditory pitch and visual position. All three types are interacting dimensions because, in each case, subjects experience a failure of selective attention known as Garner interference in a second member of Garner's set of converging operations, speeded classification (re auditory integral dimensions, see Grau \& Kemler Nelson, 1988; Melara \& Marks, 1990d; Wood, 1975; re configural dimensions, see Pomerantz \& Garner, 1973; re crossmodally corresponding dimensions, see Melara \& O'Brien, 1987). Separable dimensions yield no Garner interference (e.g., Garner \& Felfoldy, 1970).

Integral dimensions (e.g., pitch and loudness), historically the first to be studied (Garner \& Felfoldy, 1970), are those that yield performance gain in speeded classification whenever and however dimensions are correlated. Crosstalk between integral channels is thought to be restricted to a sensory/perceptual level of processing (see Melara \& Marks, 1990c, 1990d). Configural dimensions are those geometric dimensions (e.g., height and width) whose interaction can create a third, emergent dimension. Pairs of parentheses, each member of which can face either left or right (as shown in Figure 1A), are twovalued configural dimensions whose interaction yields the emergent dimension of shape or area. Corresponding dimensions are those whose pairing entails a meaningful correspondence. Thus, with the cross-modally corresponding dimensions of auditory pitch and visual vertical position, attributes either match semantically (e.g., high pitch and high position) or mismatch semantically (e.g., low pitch and high position).

\section{Previous MDS Studies}

All three types of interacting dimensions have been subjected to MDS. Melara (1989b) evaluated the similarity 

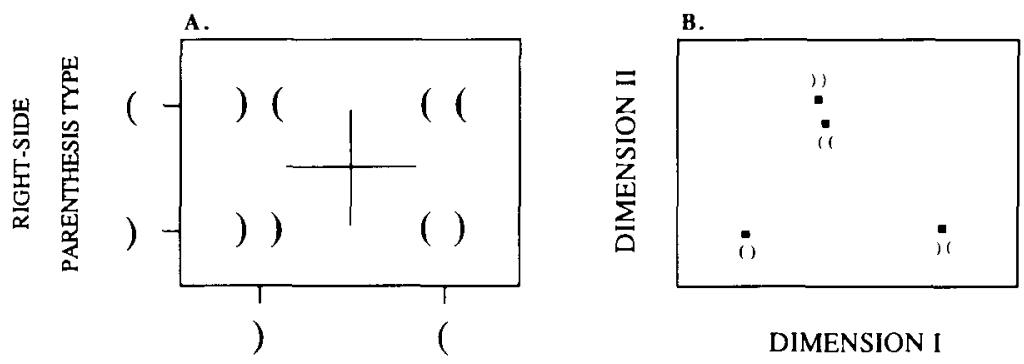

LEFT-SIDE PARENTHESIS TYPE

DIMENSION I

Figure 1. Panel A: The experimenter-defined structure for the configural dimensions of left- and right-facing parentheses. Panel B: The two-dimensional ALSCAL solution, after Lockhead and King (1977), for similarity judgments between poirs of parentheses.

relations among the cross-modally corresponding pairs of pitch-position and pitch-color. Subjects recognized (perhaps implicitly) the separate dimensions of these stimuli, as indicated by the fact that the original stimulus dimensions were always recovered. Nonetheless, both raw similarity ratings and transformed scale values in MDS were described best by the Euclidean distance function.

Lockhead and King (1977) obtained similarity ratings among stimuli formed from paired parentheses. Using alternating least squares MDS (ALSCAL; Takane, Young, \& deLeeuw, 1977), the authors obtained a good fit with a two-dimensional Euclidean solution. With configural dimensions such as these, subjects can reconstrue the stimulus set in terms of geometric properties that emerge from applying the experimenter's definition. Lockhead and King's parentheses set, for example, was defined by left- or right-positioned parentheses facing either left or right (see Figure 1A); applying this definition created an emergent dimension of symmetry-asymmetry, which could then serve as the basis of similarity judgments. Lockhead and King obtained evidence consistent with just such a redefinition, as can be seen in their scaling solution, which appears in Figure 1B: Dimension I distinguished whether stimuli were convex or concave; Dimension II distinguished whether stimuli were symmetrical or asymmetrical. ${ }^{1}$ This important finding is one that we will focus on later in this investigation.

Results with other configural dimensions (e.g., height and width of rectangles) have also shown a superiority of the Euclidean rule over the city-block rule and an effect of emergent dimensions on similarity ratings (e.g., Krantz \& Tversky, 1975; Schönemann, Dorcey, \& Kienapple, 1985; Wiener-Ehrlich, 1978). However, individual differences have also been widespread (see Schönemann, 1990). Schönemann et al. (1985), for example, found that only $43 \%$ of their subjects judged similarity according to the area and shape of rectangles; the rest relied on the experimenter-defined dimensions of height and width.

Several investigators have subjected the integral dimensions of pitch and loudness to an MDS analysis of similarity ratings, but the results have been inconclusive (e.g., Carvellas \& Schneider, 1972; Chipman \& Carey, 1975;
Zagorski, 1978). Carvellas and Schneider (1972), using magnitude estimates of dissimilarity among pure tones, were unable to find clear differences in stress between Euclidean and city-block metrics (although, on other grounds, they concluded that the city-block metric was superior). Chipman and Carey (1975) also used magnitude estimates to measure differences among bands of noise varying in frequency and intensity. Again, neither Minkowski metric was superior to the other in terms of the stress values in the MDS solution (although, on other grounds, Chipman and Carey opted for the Euclidean metric). Thus, results with interacting auditory dimensions have been ambiguous regarding the rule by which similarity is judged. In the present study, we ask whether this ambiguity can be explained in part by the role of optional processes in similarity judgments.

\section{THE PRESENT STUDY}

Subjects in this study judged the similarity of stimulus pairs under two sets of rating instructions. One set of instructions, what we shall call overall similarity instructions, emphasized judgments of the stimuli as wholes. The second set, dimensional instructions, emphasized judgments based on the component dimensions. Thus, where subjects were instructed to focus their attention-on the stimulus whole or on the stimulus dimensions-served as our manipulation of optional processes. If similarity judgments contain a large optional component, then similarity relations should change as a function of rating instructions. To evaluate the effect of these instructions on similarity structure, two different types of MDS were performed on each of the three stimulus sets-namely, pitchposition, pitch-loudness, and paired parentheses.

One analysis involved what we shall call unimatrix MDS, defined as similarity ratings averaged across subjects and scaled to optimize Kruskal's Stress Formula 1 (Kruskal, 1964). This analysis, which relies exclusively on ordinal properties in the data, was performed with the pitch-position and the pitch-loudness sets according to both the Euclidean and the city-block distance functions for one-, two-, and three-dimensional solutions. Good- 
ness of fit was evaluated by the magnitude of the stress values. A different approach, described later, was used to determine the best-fitting metric for the parentheses set.

A second analysis, the metric SINDSCAL procedure (Pruzansky, 1975; Schiffman, Reynolds, \& Young, 1981; see also Carroll \& Chang, 1970), was implemented to investigate (1) the orientation of axes in the space and (2) the prevalence of individual differences in the scaling solutions. This procedure uses individual subject information to derive what are thought to be psychologically meaningful (i.e., unique) orientations of dimensional axes (see Carroll, 1981; Carroll \& Arabie, 1980). Subject information also acts as an added constraint to inject greater determinacy into the scaling solution, a concern with small stimulus sets (Kruskal 1976, 1977). By virtue of the scaling algorithm, SINDSCAL solutions employ a Euclidean distance function. Thus, we report SINDSCAL solutions only for those data sets relatively well fit by the Euclidean metric, as determined by Kruskal's nonmetric procedure.

\section{Method}

\section{Subjects}

Eighteen young men and women ( 5 men, 13 women), mostly students from Yale University and the New School for Social Research, were paid $\$ 5$ per hour to participate.

\section{Stimuli and Apparatus}

All three sets of stimuli were generated by a Commodore 64 microcomputer. Visual stimuli were presented on either a Taxan 220 or a Super Brain CM13L color monitor. Auditory stimuli were generated by the microcomputer's SID chip; the signals were sent to a Realistic SA-150 amplifier, and presented over Realistic Nova40 headphones.

The pitch-position set comprised tones paired with visually presented crosses. The tones had complex, rectangular envelope waveforms with fundamental frequencies of 1047,611 , or $175 \mathrm{~Hz}$. Power spectra for signals like these were described in an earlier article (Melara \& Marks, 1990b). All sounds were $70 \mathrm{~dB}(\mathrm{~A})$. The crosses subtended $0.26^{\circ}$ of visual angle and appeared in either a high $\left(3.43^{\circ}\right.$ above midline), middle center, or low $\left(3.43^{\circ}\right.$ below midline) screen position. Crosses appeared in white (Commodore color No. 1) against a gray screen background (color No. 11). The stimulus set contained nine stimuli-that is, all nine combinations of the three pitches and three positions. The cross-modal stimuli were displayed and terminated simultaneously, lasting approximately $1 \mathrm{sec}$.

The pitch-loudness set comprised complex tones of 1000,950 , or $900 \mathrm{~Hz}$ and 80,70 , or $60 \mathrm{~dB}$. Intensity was calibrated on the A scale of a General Radio 1565-B sound-level meter. Duration was approximately $1 \mathrm{sec}$.

The parentheses set contained the four possible pairs of normally oriented parentheses, as shown in Figure 1A. Parentheses appeared at center screen in black (color No. 0) against a gray screen background. Parentheses subtended $0.44^{\circ}$ of visual angle and were separated by $0.35^{\circ}$. Duration was approximately $1 \mathrm{sec}$.

\section{Procedure}

The subjects were tested individually in a quiet room. Each subject participated in one session of six rating tasks that collectively lasted approximately $3 \mathrm{~h}$. The subjects could rest whenever they wished. Half of the rating tasks used overall similarity instructions, and half used dimensional instructions. For each of the three stimulus sets, the subjects first rated under one set of instructions and then the other; order of instructions and stimulus sets were counter- balanced across subjects. Overall similarity instructions for pitch and loudness were stated as follows:

On each trial, you will be presented with two tones. Your task is to rate how similar or different the pair are. The tones vary in pitch and loudness. To rate them, we want you to concentrate on the overall similarity of the sounds. Think about how similar the sounds are to each other by considering each sound as a "whole" or "Gestalt." Your rating should reflect the similarity of these two Gestalts. You may listen to the tones by pressing keys $\# 1$ and $\# 2$. Do this as often as you wish. When you are ready to rate them, simply press the space bar. Use the CRSR keys located on the lower right of the keyboard to move the arrow to the desired place on the scale. When you are finished, press RETURN to go on to the next trial.

Dimensional instructions for pitch and loudness were stated as follows:

On each trial, you will be presented with two tones. Your task is to rate how similar or different the pair are. The tones vary in pitch and loudness. To rate them, we want you to concentrate separately on their pitch and loudness. Think about how similar the sounds are to each other in pitch and in loudness. Your rating should reflect the similarity of the sounds along these dimensions. You may listen to the tones by pressing keys \#1 and \#2. Do this as often as you wish. When you are ready to rate them, simply press the space bar. Use the CRSR keys located on the lower right of the keyboard to move the arrow to the desired place on the scale. When you are finished, press RETURN to go on to the next trial.

Analogous instructions were created for the cross-modal stimuli and the parentheses stimuli.

Each task began by familiarizing the subjects with the stimulus set. The nine pitch-loudness stimuli were presented in a random order, as often as desired by the subject. The three sounds in the pitch-position set were sequenced by decreasing pitch. In the rating task proper, stimuli were presented in pairs; each stimulus was under the subject's control and could be presented as often as desired within a trial. The particular pair appearing in a given trial was determined by random selection. Included were pairs in which the two stimuli were identical. In the pitch-loudness and pitch-position sets, which included nine stimuli each, the $\mathbf{4 5}$ possible pairs were presented twice during the task, yielding 90 total trials. In the parentheses set, which included only four stimuli, the 10 possible pairs were presented four times, yielding 40 total trials. To rate a pair of stimuli, the subjects pressed the keyboard space bar and a computer-generated rating scale appeared. The rightmost pole of the scale was labeled very different; the leftmost pole was labeled very similar. Ratings were made by positioning a diamond-shaped marker on the scale to one of $\mathbf{4 0}$ possible points and pressing the carriage return. The next trial followed immediately after the subject's response.

\section{Results and Discussion}

\section{Pitch-Position Stimulus Set}

Unimatrix MDS solutions. A single triangular matrix, created by averaging ratings across subjects, was used as input in each of four unimatrix MDS analyses. ${ }^{2}$ Unimatrix analyses were performed twice on ratings from each type of instructions, once fitting the Euclidean distance function and once fitting the city-block distance function. Solutions were obtained in one, two, and three dimensions. The stress values (optimizing Kruskal's Stress Formula 1) for these analyses appear in Table 1.

In one dimension, Euclidean and city-block metrics are equivalent mathematically; thus, the stress values are identical. The fit with one-dimensional solutions is poor, having stress values of .371 and .313 for overall similarity 
Table 1

Stress Values of One-, Two-, and Three-Dimensional Nonmetric Solutions, Fitting Euclidean and City-Block Distance Functions, for Ratings of Pitch-Position Stimuli Under Overall Similarity and Dimensional Instructions

\begin{tabular}{lcccccccc}
\hline & \multicolumn{3}{c}{ Euclidean Metric } & & \multicolumn{3}{c}{ City-Block Metric } \\
\cline { 2 - 3 } \cline { 7 - 8 } Rating Instructions & \multicolumn{3}{c}{ Dimensionality } & & \multicolumn{3}{c}{ Dimensionality } \\
\cline { 2 - 3 } \cline { 6 - 8 } & One & Two & Three & & One & Two & Three \\
\hline Overall Similarity & .371 & .111 & .008 & & .371 & .187 & .046 \\
Dimensional & .313 & .104 & .024 & & .313 & .022 & .011 \\
\hline
\end{tabular}

instructions and dimensional instructions, respectively; so we believe that it is not an appropriate dimensionality for these data sets.

The two- and three-dimensional solutions show clear differences between the Euclidean and city-block metrics as a function of rating instructions. When the subjects rated stimuli under overall similarity instructions, scale values were fit better by the Euclidean metric than by the cityblock metric, regardless of dimensionality. This result accords with previous MDS analyses involving cross-modally corresponding dimensions (Melara, 1989b). However, unlike Melara's (1989b) result, when the subjects rated stimuli under dimensional instructions, scale values were fit better by the city-block metric than by the Euclidean metric, again regardless of the solution's dimensionality.

At least with these interacting dimensions, then, the distance rule used by subjects to determine similarity is influenced heavily by what subjects are told to attend tonamely, the stimulus whole or the stimulus dimensions. As one can see in Figure 2A, which depicts stress values obtained in the two-dimensional solutions, the instructional manipulation had its greatest effect on city-block goodness of fit: This metric fared well under dimensional instructions, but very poorly under overall similarity instructions. The Euclidean metric fit the data about equally well under the two sets of instructions. More generally, Figure 2A shows that simple changes in rating instruc-

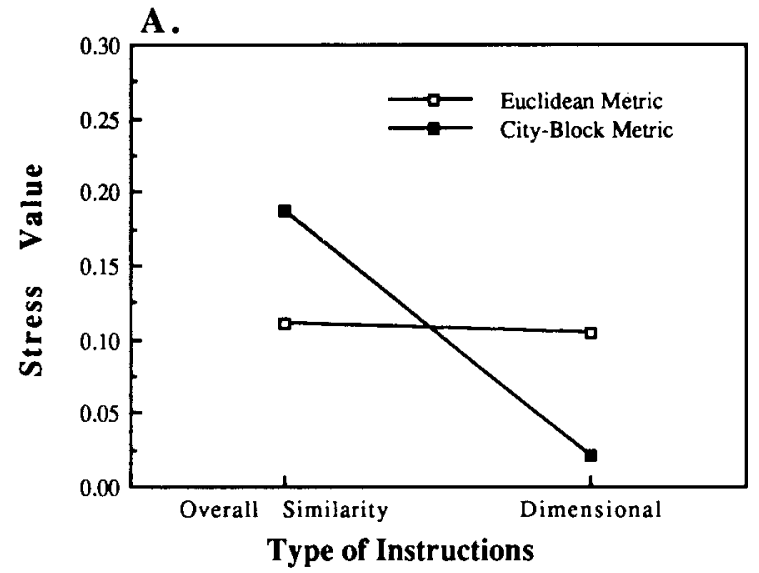

tions can clearly affect which of two metrics yields the better fit in similarity scaling.

SINDSCAL solutions. SINDSCAL is a metric procedure that implements a Euclidean distance function. The unimatrix analyses reported above indicate that, when comparing a Euclidean fit with a city-block fit to ratings of pitch-position stimuli, Euclidean is the best-fitting metric only under overall similarity instructions. Consequently, we report only the SINDSCAL analysis performed on these data.

For each subject under overall similarity instructions, a triangular matrix of similarity ratings was created by averaging across replications. Thus, 18 matrices served as input for a single SINDSCAL analysis. A geometric depiction of the two-dimensional solution appears in Figure $3 .{ }^{3}$ As one can see, Dimension I (33\% variance) corresponds closely to pitch, whereas Dimension II (30\% variance) appears to be position. A very similar solution, with respect both to dimensionality and to the relative location of stimulus points, obtained when fitting a cityblock metric in unimatrix analyses of ratings made under dimensional instructions. The close agreement between the SINDSCAL and unimatrix analyses underscores the psychological importance of pitch and position in subjects' judgments of similarity. The weight matrix in the SINDSCAL solution, which contains weightings of each subject on each dimension, indicated good agreement among subjects on both dimensions. Weights exceeding .30 were obtained for 17 of 18 subjects on Dimension I and for 16 of 18 subjects on Dimension II. Apparently, then, subjects acknowledge the experimenter-defined dimensions of pitch and position when making overall similarity judgments of cross-modal stimuli. Importantly, these weightings were not constrained by homogeneity (cf. Wish \& Carroll, 1974), because weightings on each dimension were appropriately variable, having a range of .92 on Dimension $I(M=.54, \sigma=.20)$ and .76 on Dimension II $(M=.52, \sigma=.20)$.

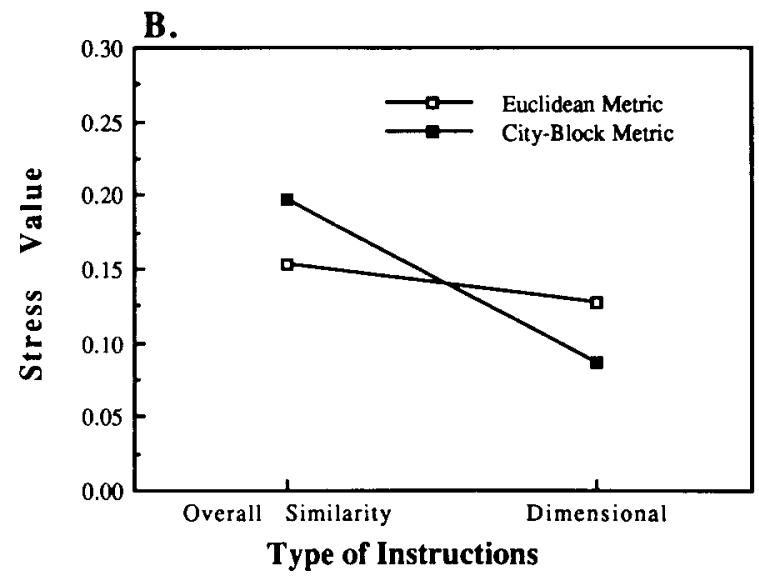

Figure 2. Stress values to ratings of pitch-position stimuli (panel A) and pitch-loudness stimuli (panel B) under overall similarity instructions and dimensional instructions, obtained in two-dimensional Kruskal solutions for Euclidean and city-block metrics. 


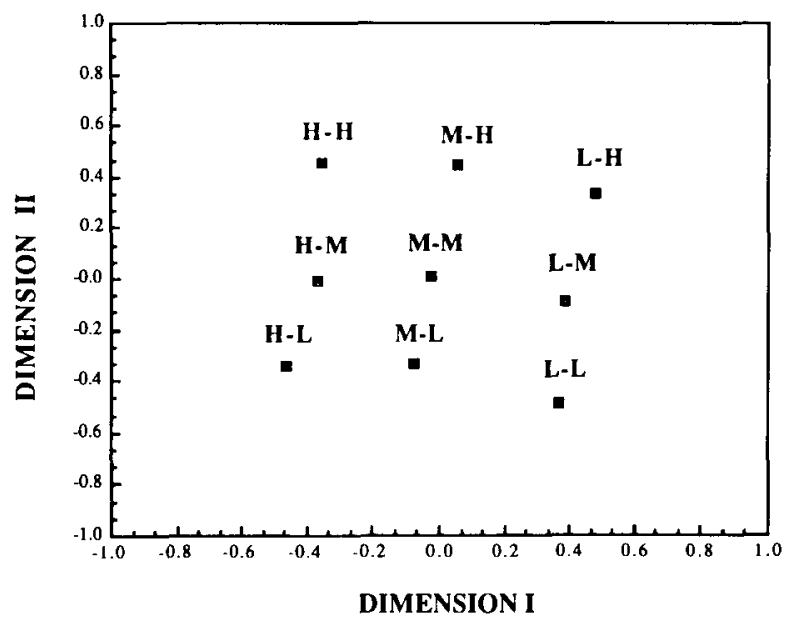

Figure 3. The two-dimensional SINDSCAL solution for similarity judgments of pitch-position stimuli, rated according to overall similarity instructions. Note-In the abbreviations, the first letter indicates frequency $(H=h i g h, M=$ middle, $L=l o w)$ and the second letter indicates vertical position (H $=$ high, $M=$ middle, $L=$ low).

Table 2

Stress Values of One-, Two-, and Three-Dimensional Nonmetric Solutions, Fitting Euclidean and City-Block Distance Functions, for Ratings of Pitch-Loudness Stimuli Under Overall Similarity and Dimensional Instructions

\begin{tabular}{lccccccc}
\hline & \multicolumn{3}{c}{ Euclidean Metric } & & \multicolumn{3}{c}{ City-Block Metric } \\
\cline { 2 - 4 } \cline { 6 - 8 } Rating Instructions & One & Two & Three & & One & Two & Three \\
\cline { 2 - 4 } & Ovensionality & & \multicolumn{3}{c}{ Dimensionality } \\
\hline Overall Similarity & .355 & .154 & .001 & & .355 & .197 & .023 \\
Dimensional & .310 & .127 & .001 & & .310 & .086 & .003 \\
\hline
\end{tabular}

\section{Pitch-Loudness Stimulus Set}

Unimatrix MDS solutions. One-, two-, and threedimensional solutions for ratings obtained under overall similarity and dimensional instructions, and fitted with both Euclidean and city-block functions, appear in Table 2 . The results resemble those obtained with pitchposition. The one-dimensional fits are poor and therefore are not considered viable solutions for either set of ratings. The fits obtained in two dimensions, and to a lesser extent in three dimensions, are dictated by a match between instructions and metric. With overall similarity instructions, the Euclidean metric provides the better fit, both in two dimensions and in three dimensions. However, with dimensional instructions, the city-block metric is superior, at least in the two-dimensional case. ${ }^{4}$

As was found with pitch-position, examination of Figure $2 \mathrm{~B}$ reveals that it is the city-block metric that was affected most by the change in instructions; relative to the Euclidean metric, stress values of the city-block metric changed substantially between the two types of instructions. More generally, Figure 2B indicates that the appropriate metric of pitch-loudness similarity apparently has less to do with what stimuli or what measure was used than it does with to what information subjects attend when making judgments: the stimulus whole or the stimulus dimensions.

SINDSCAL solutions. Unimatrix analyses indicated that only judgments obtained under overall similarity instructions are fit better by the Euclidean metric than by the city-block metric; thus, only these ratings were subjected to SINDSCAL. The two-dimensional SINDSCAL solution appears in Figure 4. This solution accounted for $62 \%$ of total variance. Dimension I ( $32 \%$ variance) corresponds closely to pitch; Dimension II (30\% variance) corresponds to loudness. As with the pitch-position stimuli, both the dimensions and the relative stimulus configuration correspond well to those obtained in a city-block fit by unimatrix analyses of ratings made under dimensional instructions. Agreement among subjects was good: Examination of the weight matrix revealed weights greater than .30 for 17 of 18 subjects on Dimension I and for 15 of 18 subjects on Dimension II. So, as with pitch-position, overall similarity instructions do not hinder access to the underlying stimulus dimensions. And, as with pitchposition, weightings were variable, yielding a range of .50 on Dimension $I(M=.55, \sigma=.14)$ and .83 on Dimension II $(M=.51, \sigma=.05)$.

\section{Parentheses Stimulus Set}

Best-fitting metrics. With only four stimuli in the set, too few constraints exist to derive unimatrix MDS solutions that are not degenerate (see Kruskal, 1976). As an alternative way to determine the appropriate distance functions, a comparison was made between the observed dissimilarities among stimuli that differ on both dimensions (i.e., the hypotenuses of the experimenter-defined space) and the predicted dissimilarities using Euclidean and cityblock power metrics (see Melara, 1989b, for more details). The results of this comparison appear in Figures 5A (overall similarity instructions) and 5B (dimensional instructions).

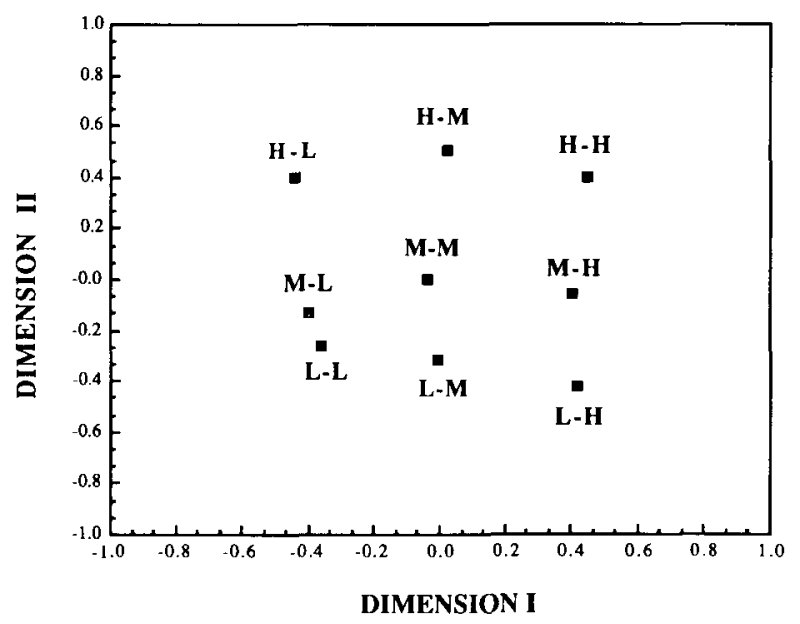

Figure 4. The two-dimensional SINDSCAL solution for similarity judgments of pitch-loudness stimuli, rated according to overall similarity instructions. Note - In the abbreviations, the first letter indicates frequency ( $H=$ high, $M=$ middle, $L=$ low) and the second letter indicates intensity $(H=$ high, $M=$ middle, $L=$ low). 
A.

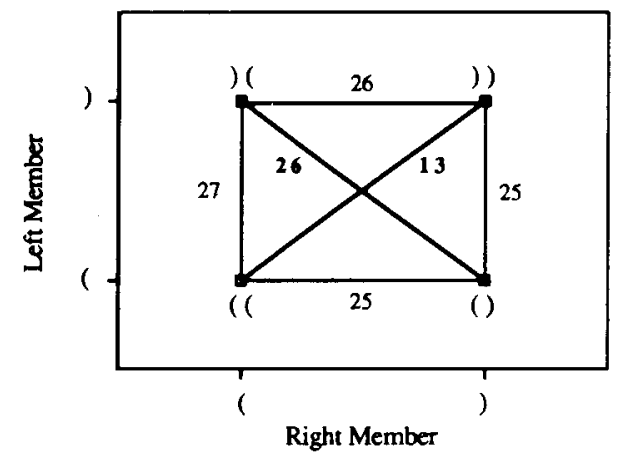

PREDICTED BIDIMENSIONALDISSIMILARITY
B.

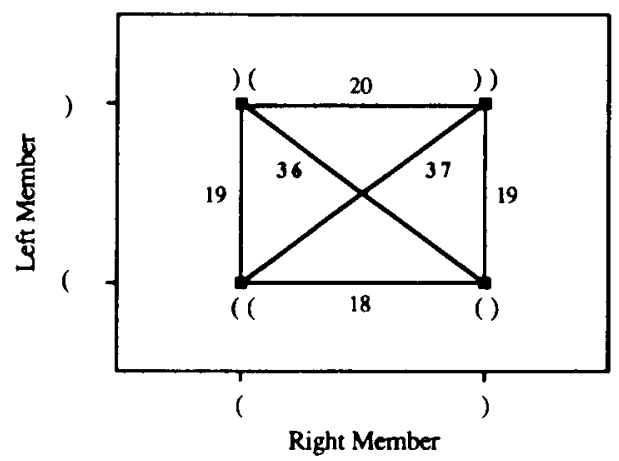

PREDICTED BIDIMENSIONAL DISSIMIILARITY
Euclidean metric:
26.9
City-block metric: $\quad 38.0$
Estimated r:
1.06

Figure 5. Rated dissimilarities of parentheses stimuli, along with predicted bidimensional dissimilarities derived from the Euclidean and city-block metrics, for ratings made according to either overall similarity instructions (panel A) or dimensional instructions (panel B). Note-Observed bidimensional dissimilarities are highlighted alongside the diagonals.

With overall similarity instructions, the hypotenuses are not fit well by either the Euclidean or the city-block metric. Nonetheless, it is clear that the untransformed dissimilarities are closer to what would be expected by a Euclidean distance function. Using Tversky and Gati's (1982) equation for estimating the Minkowski $r$ exponent, ${ }^{5}$ we obtained a value of $r=2.5$. This indicates that the appropriate distance function is better approximated by a Euclidean metric $(r=2.0)$ than by a city-block metric $(r=1.0)$.

With dimensional instructions, a clear superiority was found with the city-block metric compared with the Euclidean metric. As can be seen in Figure 5B, the dissimilarities along the hypotenuses are well predicted by the city-block function. Moreover, the Minkowski value was estimated to be $r=1.06$, very close to the city-block exponent of $r=1.0$. Thus, the results with parentheses stimuli are compatible with results obtained using other stimulus sets. Specifically, the best-fitting distance function in similarity space depends on how subjects attend to stimulus dimensions.

SINDSCAL solutions. Unlike the distance function estimates made to stimuli in the pitch-position and pitchloudness sets, which emerged from nonmetric analyses, our estimates for the parentheses stimuli are based in analyses that assume metric properties in the data (e.g., ratiolevel scale of measurement of differences in similarity; see Beals, Krantz, \& Tversky, 1968). With the earlier stimulus sets, the nonmetric analyses provided us with a rationale for deciding which data sets to subject to the metric SINDSCAL procedure. Because metric analyses were used with the parentheses stimuli, we have no such rationale in the case of these data sets. To avoid being arbitrary, we decided to obtain two-dimensional SINDSCAL solutions for ratings made under both overall similarity instructions and dimensional instructions. These appear in Figures 6A and 6B, respectively. A reasonable linear relationship was found between the six interstimulus distances obtained in each SINDSCAL solution and the corresponding averaged ratings of stimulus similarity (Pearson $r=$ -.53 , with overall similarity instructions, and $r=-.61$, with dimensional instructions, with little evidence of nonlinearity); thus, our assumption that the differences in similarity are ratio scaled seems reasonable, at least as a first approximation (but see Parker, Casey, Ziriax, \& Silberberg, 1988).

With overall similarity instructions, the solution accounted for $80 \%$ of total variance in the rating data. Notice the striking resemblance between this solution (Figure 6A) and Lockhead and King's (1977) ALSCAL solution for the same stimuli (Figure 1B). As they concluded, Dimension I (41\% variance) appears to be one of concavityconvexity, whereas Dimension II (39\% variance) is one of symmetry-asymmetry (see also Treisman, 1986).

That this is not the only possible similarity structure is revealed by the SINDSCAL solution to ratings obtained with dimensional instructions (Figure 6B). This latter solution accounted for $90 \%$ of total variance. Notice the similarity between this solution and the original experimenterdefined stimulus axes (Figure 1A). Extrapolating from that stimulus space, Dimension I ( $49 \%$ variance) can be construed as left-parenthesis type, but rotated clockwise $45^{\circ}$; Dimension II can be construed as right-parenthesis type, rotated clockwise $45^{\circ}$. Of course, this interpretation assumes that SINDSCAL solutions are not dimensionally unique. ${ }^{6}$ When the orientation given in the solution is used 

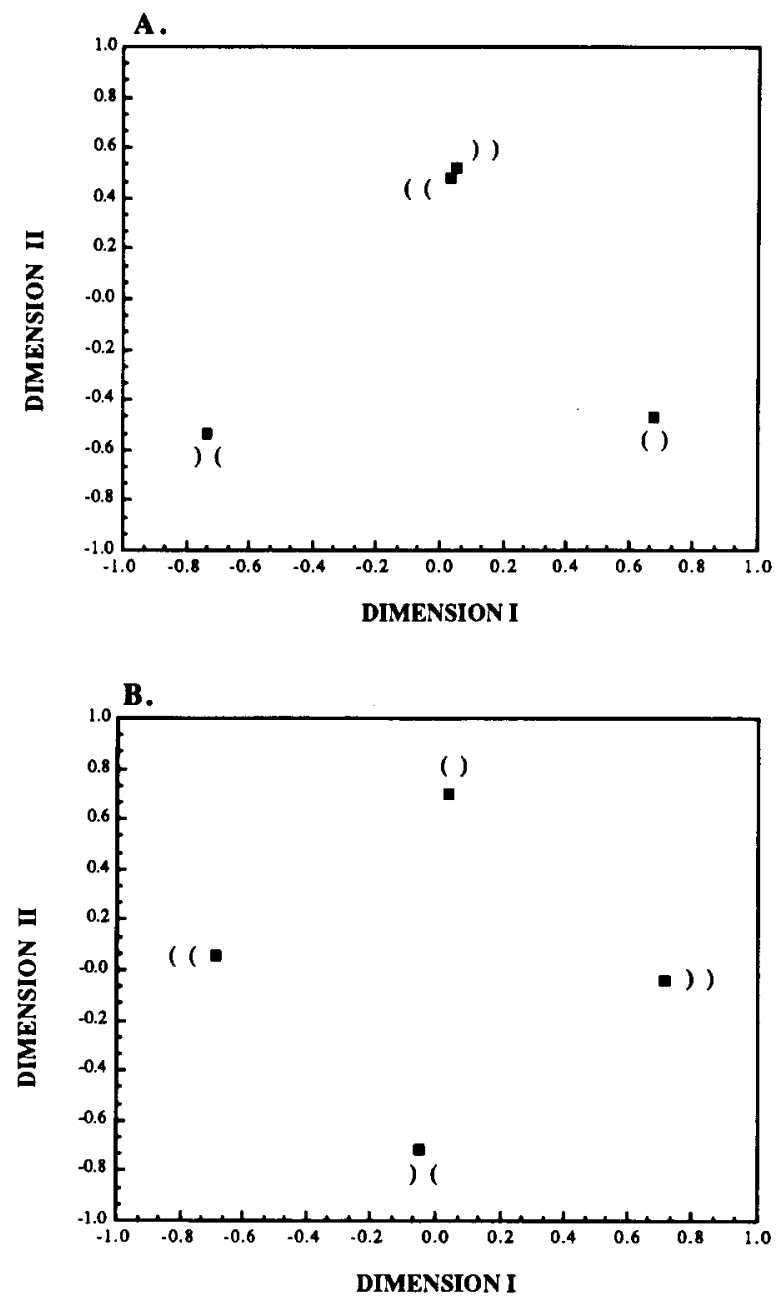

Figure 6. The two-dimensional SINDSCAL solution for similarity judgments of parentheses stimuli, rated according to either overall similarity instructions (panel A) or dimensional instructions (panel B).

instead, one can interpret Dimension I as the degree to which the stimulus faces left or faces right and Dimension II as the degree of convexity-concavity, although this interpretation seems less consistent with the instructional set. In any case, the SINDSCAL solutions to parentheses stimuli confirm that the structure of the stimulus space changes depending on where attention is directed, to the whole or to the dimensions.

\section{GENERAL DISCUSSION}

In this study, we analyzed similarity judgments to stimuli formed from three different pairs of interacting dimensions: (1) integral dimensions-auditory pitch and loudness; (2) configural dimensions-pairs of parentheses; (3) corresponding dimensions-auditory pitch and visual position. The subjects rated similarity among stimuli within each of these three sets according to two types of instruc- tions. Overall similarity instructions encouraged the subjects to consider variation along the pair of dimensions together (i.e., as a whole). For all three pairs of interacting dimensions, attending to the stimuli as wholes led to similarity judgments structured better by a Euclidean distance function than by a city-block distance function. Dimensional instructions encouraged the subjects to consider variation along the pair of dimensions separately (i.e., one dimension at a time). For all three pairs of interacting dimensions, attending to stimulus dimensions led to similarity judgments whose structure was described better by a city-block distance function than by a Euclidean function. Thus, for all known forms of dimensional interaction, the structure of the similarity space is determined largely by where subjects focus their attention. We now discuss several implications of these findings.

\section{Investigating Similarity Relations}

The present results show clear limitations on the use of direct measures of stimulus similarity, whether ratings or magnitude estimates, to investigate the nature of psychological similarity. It is not particularly surprising that subjects can attend either to the stimulus whole or to its constituent dimensions, even when stimulus dimensions interact. Indeed, Garner (1974b) has stressed the dual character of stimuli formed from integral dimensions such as saturation and brightness. A color, for example, can be regarded either as a whole or as a set of values on separate dimensions (e.g., hue, saturation, and brightness, or redness-greenness, blueness-yellowness, and brightness-darkness). What is surprising is that simple changes in instructions, such as the differences between our overall similarity instructions and our dimensional instructions, result in such profound changes in the similarity space.

One is forced to conclude that whatever mandatory processes underlie psychological similarity among multidimensional stimuli, these processes may be measured only poorly by direct judgments. Moreover, to the extent that direct measures, such as ratings, have been shown to correlate highly with indirect measures, such as same-different reaction times (see, e.g., Melara, 1989b; Podgorny \& Garner, 1979), one can conclude that these indirect measures may be no better at gauging mandatory processes. In both cases, optional processes can obscure identification of mandatory components.

Two important considerations limit our conclusions: (1) the stimuli and dimensions we tested and (2) the metrics we fitted. First, the results reported here concern similarity relations among multidimensional stimuli, because only when multiple dimensions are involved is it meaningful to speak of a combination rule. Thus, the optional processes uncovered in this study are presumably not present in unidimensional judgments of similarity. Further research is needed to evaluate how unidimensional judgments may be influenced by other optional processes (cf. Parker et al., 1975). Moreover, we tested only interacting dimensions, and so our conclusions may not generalize to separable dimensions. We suspect, though, 
that similarity judgments of separable dimensions are, if anything, even more prone to the influence of optional processes, because values on these dimensions are not integrated mandatorily. Nonetheless, future research will need to evaluate this suspicion.

Second, although we found that instructions affect what combination rule subjects adopt, this does not imply necessarily that any rule is possible given appropriate instructions. Neither do our results imply that only the city-block and Euclidean metrics can be isolated through instructional manipulations. Presumably, other metrics of similarity can be recovered, but this pool is likely to be small. For example, one can imagine that appropriate instructions might allow the supremum metric (Minkowski $r=\infty$ ) to emerge, where the dimension having the largest change determines similarity judgments. Likewise, it might be possible to obtain reliable Minkowski metrics between $r=1$ and $r=2$, or even distance functions unrelated to the Minkowski family (see, e.g., Schönemann, 1990; Schönemann et al., 1985). However, usable distance functions are almost certainly limited by psychological plausibility: It is unlikely that instructions could be devised that yield distance functions chosen arbitrarily and having no psychological realization. In this respect, there appear to be mandatory constraints on the psychologically possible ways of conceptualizing multidimensional similarity relations.

\section{When Optional Processes Are Found}

Clearly, the presence of optional processes, such as those found here, can undermine claims about the nature of underlying cognitive representations. When optional processes are found, the researcher should suspect and reconsider three broad classes of conclusion. First, the researcher must suspect measurement validity, because the measures may not be gauging the intended psychological processes. In the present study, we were led to question the idea that by identifying the metric that fits a set of similarity ratings best, one specifies a single rule of combination.

Second, optional processes make it more difficult to identify fundamental properties of processing. In the present study, we were led to question the primacy of emergent dimensions in configural interaction (cf. Pomerantz, 1986; Treisman \& Paterson, 1984), because, with dimensional instructions, subjects could effectively ignore these dimensions. We were also unable to determine whether, in the mandatory sense, interacting dimensions are processed as a perceptual blob (e.g., Lockhead, 1979)—as would be expected by a Euclidean model-or are processed independently (e.g., Zagorski, 1975, 1978)-as would be expected by a city-block model. Our results indicate that this issue will need to await a different measure of psychological similarity, one not so prone to the effects of optional processes.

Finally, the presence of optional processes raises questions about the psychological "law." In particular, the present findings lead one to question Shepard's (1987) law of generalization, because no longer can it be claimed with certainty that interacting dimensions follow a Euclidean distance function and that separable dimensions follow a city-block distance function. In our view, psychological laws, when they are discovered, probably will lie in an understanding of mandatory processes. And mandatory processes can only be uncovered by first identifying and isolating the optional processes that contextualize them.

\section{REFERENCES}

Attneave, F. (1950). Dimensions of similarity. American Joumal of Psychology, 63, 516-556.

Benls, R., Krantz, D. H., \& Tversky, A. (1968). The foundations of multidimensional scaling. Psychological Review, 75, 127-142.

CARrol.L, J. D. (1981). INDSCAL. In S. S. Schiffman, M. L. Reynolds, \& F. W. Young (Eds.), Introduction to multidimensional scaling (pp. 371-389). New York: Academic Press.

CARroll, J. D., a ARABIR, P. (1980). Multidimensional scaling. Annual Review of Psychology, 31, 607-649.

Carroll, J. D., * Chang, J. J. (1970). Analysis of individual differences in multidimensional scaling via an $n$-way generalization of "Eckard-Young" decomposition. Psychometrika, 35, 283-319.

Carvellas, T., Schneider, B. (1972). Direct estimation of multidimensional tonal dissimilarity. Joumal of the Acoustical Society of America, 51, 1839-1848.

Chipman, S. F., \& Carey, S. (1975). Anatomy of a stimulus domain: The relation between multidimensional and unidimensional scaling of noise bands. Perception \& Psychophysics, 17, 417-424.

ENNIS, D. M. (1988). Technical comment: Toward a universal law of generalization. Science, 242, 944.

ENNIS, D. M., PALEN, J. J., MULleN, K. (1988). A multidimensional stochastic theory of similarity. Journal of Mathematical Psychology, $32,449-465$.

Fraser, C. O. (1976). Cognitive strategies and multidimensional scaling. British Journal of Psychology, 67, 399-406.

GARNER, W. R. (1974a). Attention: The processing of multiple sources of information. In E. C. Carterette \& M. P. Friedman (Eds.), Handbook of perception (Vol. 2, pp. 23-59). New York: Academic Press.

GARNER, W. R. (1974b). The processing of information and structure. Potomac, MD: Erlbaum.

Garner, W. R., Felfoldy, G. L. (1970). Integrality of stimulus dimensions in various types of information processing. Cognitive Psychology, 1, 225-241.

Garner, W. R., Hake, H. W., \& Eriksen, C. W. (1956). Operationism and the concept of perception. Psychological Review, 63, 49-159.

Grau, J. W., Kemler Nelson, D. G. (1988). The distinction between integral and separable dimensions: Evidence for the integrality of pitch and loudness. Joumal of Experimental Psychology: General, 117, 347-370.

Handel, S., ImAI, S. (1972). The free classification of analyzable and unanalyzable stimuli. Perception \& Psychophysics, 12, 108-116.

Hyman, R., WELL, A. (1967). Judgments of similarity and spatial models. Perception \& Psychophysics, 2, 233-248.

Hyman, R., Well, A. (1968). Perceptual separability and spatial models. Perception \& Psychophysics, 3, 161-165.

InDOW, T., KANAzAWA, K. (1960). Multidimensional mapping of Munsell colors varying in hue, chroma, and value. Joumal of Experimental Psychology, 59, 330-336.

KRANTZ, D., \& TVERSKY, A. (1975). Similarity of rectangles: An analysis of subjective dimensions. Joumal of Mathematical Psychology, 12, 4-34.

KRUSKAL, J. B. (1964). Multidimensional scaling by optimizing goodness of fit to a nonmetric hypothesis. Psychometrika, 29, 1-27.

KRUSKAL, J. B. (1976). More factors than subjects, tests and treatments: An indeterminacy theorem for canonical decomposition and individual differences scaling. Psychometrika, 41, 281-293. 
KruSKal, J. B. (1977). Three-way arrays: Rank and uniqueness of trilinear decompositions, with application to arithmetic complexity and statistics. Linear Algebra \& Its Applications, 18, 95-138.

LOCKHEAD, G. R. (1979). Holistic versus analytic process models: A reply. Joumal of Experimental Psychology: Human Perception \& Performance, 5, 746-755.

LOCKHEAD, G. R., \&ING, M. C. (1977). Classifying integral stimuli. Journal of Experimental Psychology: Human Perception \& Performance, 3, 436-443.

MARKs, L. E. (1987). On cross-modal similarity: Perceiving temporal patterns by hearing, touch, and vision. Perception \& Psychophysics, 42, 250-256.

MelarA, R. D. (1989a). Dimensional interaction between color and pitch. Joumal of Experimental Psychology: Human Perception \& Performance, 15, 69-79.

MelARA, R. D. (1989b). Similarity relations among synesthetic stimuli and their attributes. Journal of Experimental Psychology: Human Perception \& Performance, 115, 212-231.

MelARA, R. D. (1990, November). Mandatory processes: Consider your options. Paper presented at the 31 st Annual Meeting of the Psychonomic Society, New Orleans, LA.

Melara, R. D., Marks, L. E. (1990a). HARD and soft interacting dimensions: Differential effects of dual context on classification. Perception \& Psychophysics, 47, 307-325.

MelARA, R. D., \& MARKs, L. E. (1990b). Interaction among auditory dimensions: Timbre, pitch, and loudness. Perception \& Psychophysics, 48, 169-178.

Mrlara, R. D., Marks, L. E. (1990c). Perceptual primacy of dimensions: Support for a model of dimensional interaction. Journal of $E x$ perimental Psychology: Human Perception \& Performance, 16, 398-414.

Mrlara, R. D., Marks, L. E. (1990d). Processes underlying dimensional interactions: Correspondences between linguistic and nonlinguistic dimensions. Memory \& Cognition, 18, 477-495.

Melara, R. D., * O'Brien, T. P. (1987). Interaction between synesthetically corresponding dimensions. Journal of Experimental Psychology: General, 116, 323-336.

Parker, S., Casey, J., Ziriax, J. M., \& Silberberg, A. (1988). Random monotone data fit simple algebraic models: Correlation is not confirmation. Psychological Bulletin, 104, 417-423.

PARker, S., Schneider, B., KANow, G. (1975). Ratio scale measurement of the perceived length of lines. Journal of Experimental Psychology: Human Perception \& Performance, 104, 195-204.

Podgorny, P., \& GARNer, W. R. (1979). Reaction time as a measure of inter- and intraobject visual similarity: Letters of the alphabet. Perception \& Psychophysics, 26, 37-52.

Pomrrantz, J. R. (1986). Visual form perception: An overview. In E. C. Schwab \& H. C. Nusbaum (Eds.), Pattern recognition by humans and machines: Visual perception (pp. 1-30). New York: Academic Press.

Pomerantz, J. R., \& Garner, W. R. (1973). Stimulus configurations in selective attention tasks. Perception \& Psychophysics, 14, 565-569.

Pruzansky, S. (1975). How to use SINDSCAL: A computer program for individual differences in multidimensional scaling. Murray Hill, NJ: Bell Telephone Laboratories.

Schiffman, S. S., ReYnolds, M. L., \& Young, F. W. (1981). Introduction to multidimensional scaling. New York: Academic Press.

SCHönemanN, P. H. (1990). Psychophysical maps for rectangles. In H.-G. Geissler (Ed.), Psychophysical explorations of mental structures (pp. 149-164). Toronto, Canada: Hogrefe \& Huber.

Schönemann, P. H., DoRCey, T., KIEnAPPLE, K. (1985). Subadditive concatenation in dissimilarity judgments. Perception \& Psychophysics, 38, 1-17.

SHEPARD, R. N. (1964). Attention and the metric structure of the stimulus space. Journal of Mathematical Psychology, 1, 54-87.

SHEPARD, R. N. (1987). Toward a universal low of generalization for psychological science. Science, 237, 1317-1323.

Shepard, R. N., Kilpatric, D. W., Cunningham, J. P. (1975). The internal representation of numbers. Cognitive Psychology, 7 , 82-138.
SMith, L. B., \& KemLER, D. G. (1978). Levels of experienced dimensionality in children and adults. Cognitive Psychology, 10, 502-532.

Takane, Y., Young, F. W., deleeuw, J. (1977). Nonmetric individual differences multidimensional scaling: An alternating least squares method with optimal scaling features. Psychometrika, 42, 7-67.

Torgerson, W. S. (1958). Theory and methods of scaling. New York: Wiley.

Treisman, A. M. (1986). Properties, parts and objects. In K. R. Boff, L. Kaufman, \& J. P. Thomas (Eds.), Handbook of perception and human performance (Vol. 2, chap. 35, pp. 1-70). New York: Wiley. Treisman, A., \& Paterson, R. (1984). Emergent features, attention, and object perception. Journal of Experimental Psychology: Human Perception \& Performance, 10, 12-31.

TVERSKY, A., GATI, I. (1982). Similarity, separability and the triangle inequality. Psychological Review, 89, 123-154.

WIENER-EHRLICH, W. K. (1978). Dimensional and metric structures in multidimensional stimuli. Perception \& Psychophysics, 24, 399-414.

Wish, M., \& CArroll, J. D. (1974). Applications of individual differences scaling to studies of human perception and judgment. In E. C. Carterette \& M. P. Friedman (Eds.), Handbook of perception (Vol. 2, pp. 449-491). New York: Academic Press.

Woon, C. C. (1975). Auditory and phonetic levels of processing in speech perception: Neurophysiological and information-processing analyses. Journal of Experimental Psychology: Human Perception \& Performance, 1, 3-20.

ZAGORSKI, M. (1975). Perceptual independence of pitch and loudness in a signal detection experiment: A processing model for 2ATFC (21FC) experiments. Perception \& Psychophysics, 17, 525-531.

ZAGORSKI, M. (1978). Noncombination of pitch and loudness in multidimensional scaling. Joumal of the Acoustical Society of America, 63, 273-274.

\section{NOTES}

1. The term dimension is used in two senses in this article. Often, the term refers to perceptual continua, such as pitch and loudness. The second reference, as in the present sentence, is to the results of MDS analyses. We attempt to clarify the particular meaning through sentence context.

2. In this article, we report unimatrix analyses from similarity data averaged across subjects. Alone, individual subject ratings were too weak to be used in MDS analyses. Consequently, this article does not report statistical tests on stress values obtained in unimatrix analyses of individual subjects.

3. Three-dimensional SINDSCAL solutions were also derived for both the pitch-position set and the pitch-loudness set. The third scaling dimension was typically one in which two values of a stimulus dimension were segregated from a third value. This scaling dimension has appeared and been discussed in our earlier work (Marks, 1987; Melara, 1989b). Because the nature of this third scaling dimension is irrelevant to our present concerns, and because the remaining two scaling dimensions were unaffected by the dimensionality of the solution, we shall not discuss threedimensional SINDSCAL solutions in this report. Admittedly, the total variance accounted for by three-dimensional solutions exceeded that accounted for by two-dimensional solutions, just as in unimatrix analyses stress was smaller in three dimensions than in two dimensions. Nonetheless, the central conceptual points raised in this article are captured best by two-dimensional analyses and are not counterindicated by other SINDSCAL or unimatrix solutions.

4. The failure to discriminate city-block metric from Euclidean metric in three dimensions (with dimensional instructions) probably reflects a kind of ceiling effect, with both metrics accounting successfully for virtually all the rating variance.

5. $r=\log 2 /\{\log 2+\log [($ center path/2)/corner path $]\}$.

6. A peculiar orientation was also obtained when the ratings to pitch-position stimuli made under dimensional instructions were subjected to SINDSCAL analysis. The two-dimensional solution for the pitch-position set accounted for $74 \%$ of total variance, with Dimension I being position rotated clockwise approximately $45^{\circ}$ and Dimension II being pitch rotated clockwise approximately $45^{\circ}$. In the case of crossmodal stimuli, it seems unlikely that these rotated orientations are the 
"true" (i.e., psychologically meaningful) dimensions of similarity. This conclusion seems even more cogent when one considers that subjects in this condition were told explicitly to attend to the separate dimensions of pitch and position when making similarity judgments between stimuli. Nevertheless, individual subject analyses performed by the SINDSCAL procedure showed little intersubject variation in weights for these two scaling dimensions. Indeed, perhaps this lack of interindividual variation contributed, at least in part, to the orientation of dimensions that obtained in SINDSCAL. Of course, the aberrations in scaling solution obtained with both pitch-position and parentheses ratings occurred only under dimensional instructions, which we have shown are not well fit by a Euclidean metric. Still, these results do raise questions about the dimensional uniqueness of solutions obtained in individual scaling procedures such as SINDSCAL. As stated earlier, SINDSCAL is sensitive to individual subject information; consequently, the derived solutions have fixed, and presumably meaningful, scale values. To the extent that SINDSCAL solutions are implausible psychologically, it would seem necessary to rotate scale values until they align with psychologically meaningful axes in the space.

(Manuscript received November 13, 1990; revision accepted for publication September 12, 1991.) 